Ann. Biol. anim. Bioch. Biophys., I973, 13 (2), 193-202.

\title{
PROTÉOLYSE TRYPSIQUE IN VITRO DE DIVERS ALIMENTS
}

\author{
Marie-Claire CAMUS, J.-C. LAPORTE et C. SAUTIER \\ Unité de Recherches Diététiques de l'I. N.S. E. R. M. (U⿴囗十) \\ Directeur : J. TREMolikeres \\ Hopital Bichat, \\ 170 Bd Ney, 75018 Paris
}

RÉSUMÉ

Nous avons déterminé in vitro la cinétique de protéolyse trypsique de divers aliments (blé, arachide, viande, lait, caséine, gluten de blé) par dosage de l'azote solubilisé dans l'acide perchlorique et des protons libérés, ainsi que leur teneur en inhibiteurs trypsiques. La protéolyse est plus rapide et complète pour les substrats d'origine animale, avec des valeurs décroissant dans l'ordre : caséine, lait, viande, blés durs, blés tendres, gluten, arachide, les différences entre les trois derniers étant très faibles. Après chauffage à roo ${ }^{\circ} \mathrm{C}$ pendant I 5 minutes, la protéolyse de la caséine est moindre, mais non celle du gluten. Après attaque pepsique, les différences entre substrats de même origine disparaissent, mais la supériorité de ceux d'origine animale persiste. Seuls, le blé et l'arachide contiennent des inhibiteurs trypsiques (1'arachide respectivement deux et quatre fois plus que les blés tendres et durs). La structure complexe des protéines des divers aliments semble intervenir plus que leur teneur en inhibiteurs trypsiques, en lysine et en arginine.

\section{INTRODUC'TION}

Nous avons étudié in vitro, la protéolyse trypsique de diverses protéines alimentaires d'origine végétale ou animale, incluses (blé, arachide, viande, lait) ou isolées (caséine, gluten de blé). Une étude de leur protéolyse pepsique (Camus et SAUTIE, I972) avait montré des différences surtout pendant la phase initiale. C'est pourquoi, si nous avons étudié la cinétique de la réaction avec une concentration d'enzyme voisine des valeurs physiologiques, nous en avons précisé la phase initiale avec une concentration moindre. Plusieurs de nos substrats pouvant renfermer des inhibiteurs 
trypsiques (PusztaI, I967), nous les avons recherchés et dosés. Nous avons enfin étudié l'influence d'une protéolyse pepsique et d'un traitement thermique préalables.

\section{MATÉRIEI, ET MÉTHODES}

Le tableau I donne les caractéristiques des substrats étudiés.

\section{TABLEAU I}

Substrats étudiés

\begin{tabular}{|c|c|}
\hline Substrats & $\begin{array}{r}\text { Pourcentage d'azote } \\
\text { (Méthode de Kjeldahl) }\end{array}$ \\
\hline $\begin{array}{l}\text { Blés durs : } \\
\quad \text { Variété hybride } \ldots \ldots \ldots \ldots \ldots \ldots \ldots \ldots \ldots \ldots \\
\quad \text { Variété Lakota } \ldots \ldots \ldots \ldots \ldots \ldots \ldots\end{array}$ & $\begin{array}{l}1,69 \\
1,49\end{array}$ \\
\hline $\begin{array}{l}\text { Blés tendres : } \\
\quad \text { Variété Étoile de Choisy } \ldots \ldots \ldots \ldots \ldots \ldots \\
\text { Variété Languedoc } \ldots \ldots \ldots \ldots \ldots \ldots \ldots\end{array}$ & $\begin{array}{l}1,47 \\
1,49\end{array}$ \\
\hline $\begin{array}{l}\text { Farine d'arachide délipidée à } 80 \text { p. } 100 \\
\text { brute. } \ldots \ldots \ldots \ldots \ldots \ldots \ldots \ldots \ldots \ldots \ldots \ldots \ldots \ldots \ldots \ldots \ldots \ldots \ldots \ldots\end{array}$ & $\begin{array}{l}7,69 \\
7,69\end{array}$ \\
\hline Muscle de bœuf (rond) ................ & 3,40 \\
\hline Lait pasteurisé . . . . . . . . . . . . . & 0,53 \\
\hline Caséine Hammarsten (Merck) $\ldots \ldots \ldots \ldots$ & 13,78 \\
\hline Gluten de blé tendre (Prolabo) $\ldots \ldots \ldots \ldots$ & 12,85 \\
\hline
\end{tabular}

Les blés sont broyés au moulin électrique. La viande est homogénéisée (Turrax) pendant 5 minutes à $4^{\circ} \mathrm{C}$. Les enzymes utilisées sont : la trypsine lyophilisée Armour à 3500 unités National Formulary par mg, la pepsine cristallisée Worthington à 2600 unités par mg.

\section{Dosage des inhibiteurs trypsiques (LAPORTE, 1964)}

On dose en présence du produit dans lequel on recherche un éventuel inhibiteur trypsique, la part restant active de la trypsine d'une solution de concentration connue. A concentration constante d'inhibiteur total, la concentration de trypsine active en fonction de la concentration de trypsine totale, est représentée par une hyperbole (fig. r). Une de ses asymptotes coupe l'axe de la concentration de trypsine totale en un point $\mathrm{S}$, d'abscisse $t$, qui donne la correspondance stœchiométrique entre la trypsine et l'inhibiteur. Le point de même abscisse sur la courbe a une ordonnée $a$, telle que :

$$
\mathrm{K}=\frac{a^{2}}{t-a},
$$

K étant la constante de dissociation apparente du complexe formé entre la trypsine et l'inhibiteur. La trypsine restant active est dosée par son action sur de la caséine dont on détermine la fraction 
dégradée par spectrophotométrie à $280 \mathrm{~m} \mu$, après précipitation de la fraction non dégradée. L'inhibiteur éventuel est recherché et dosé dans l'extrait aqueux du produit.

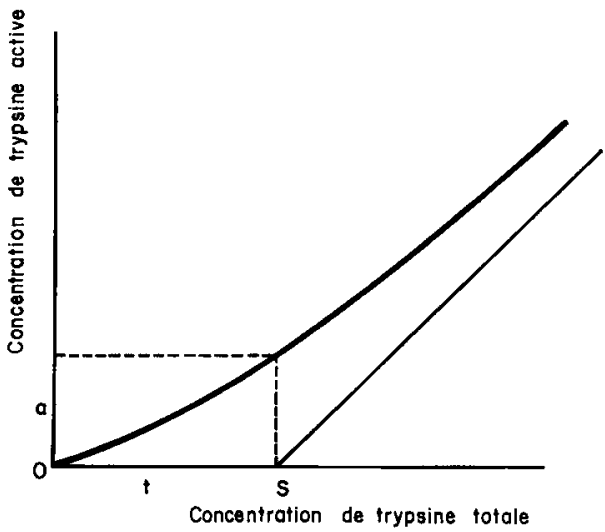

FIG. I. - Inhibition trypsique à concentration constante d'inhibiteur

\section{Protélyse trypsique}

Nous avons étudié la cinétique de protéolyse par deux méthodes :

I $^{\circ}$ dosage de l'azote solubilisé dans l'acide perchlorique.

Après l'action de l'enzyme, on sépare en les précipitant en milieu perchlorique, les protéines non attaquées des produits de dégradation, dont on dose l'azote.

$2^{\circ}$ dosage des protons libérés.

Pendant l'action de l'enzyme, on titre les protons libérés par la rupture des liaisons peptidiques.

L'étude a porté sur les produits crus, ainsi que sur la caséine et le gluten chauffés en milieu aqueux pendant $I_{5}$ minutes à $100^{\circ} \mathrm{C}$.

\section{Dosage de l'azote solubilisé dans l'acide perchlorique.}

Les divers produits sont mis en solution ou en suspension en milieu tampon phosphate de $\mathrm{Na}$, $\mathrm{pH}=7,0,0,05 \mathrm{M}$ en concentration finale. Pour un vólume de $3 \mathrm{ml}$, la concentration de substrat est $2,66 \mathrm{mg} / \mathrm{ml}$ en azote et celle de trypsine $0,66 \mathrm{mg} / \mathrm{ml}$. La réaction a lieu à $37^{\circ} \mathrm{C}$, en tubesà essai, sous agitation magnétique. Aux temps, o, 5, 10, 15, 30, 60, 120 minutes, on ajoute 4 volumes d'HClO $_{4}$ 2,5 p. Ioo, filtre et dose l'azote du filtrat par la méthode de Kjeldahl.

\section{Dosage des protons libérés (YoN, 1963).}

Les produits sont mis en solution ou en suspension aqueuse à $37^{\circ} \mathrm{C}$ dans un erlenmeyer où plonge l'électrode combinée d'un pHmètre E. I. L. sensible à ro-3 unités $\mathrm{pH}$. Sous agitation magnétique, après avoir ajusté le pH à 7,0 , on ajoute l'enzyme que l'on neutralise par $\mathrm{NaOH}$. On mesure ensuite le temps nécessaire pour revenir au $\mathrm{pH}$ initial, après adjonction d'une quantité de $\mathrm{NaOH}$ $\mathrm{o}, \mathrm{I} \mathrm{N}$, entraînant une augmentation de $\mathrm{pH}$ de 0,020 à 0,030 unité. Cette adjonction est renouvelée pendant 5 minutes environ avec une microseringue Agla. $(5 \mu \mathrm{l})$ ou avec des micropipettes Eppendorf $(20-50 \mu \mathrm{l})$. Pour un volume de $25 \mathrm{ml}$, la concentration de substrat est $2,0 \mathrm{mg} / \mathrm{ml}$ en azote et celle de trypsine $0,04 \mathrm{mg} / \mathrm{ml}$. Dans le cas d'une protéolyse pepsique préalable à $\mathrm{pH}=1,9$, celle-ci est arrêtée en ramenant le $\mathrm{pH}$ à 7,0 , à un stade correspondant à $45 \mathrm{p}$. roo d'azote solubilisé dans l'acide perchlorique.

On trace la courbe de la quantité de protons libérés en fonction du temps et sa tangente à l'origine dont la pente représente la vitesse initiale de la réaction. 


\section{RÉSULTATS}

Le tableau 2 donne la teneur en inhibiteurs trypsiques des divers substrats et la constante de dissociation apparente des complexes trypsine-inhibiteur. On constate que parmi les substrats étudiés, seuls ceux d'origine végétale renferment des inhibiteurs, sauf le gluten. La teneur des blés tendres en est deux fois plus élevée que celle des blés durs. Rapportée à l'azote, celle de l'arachide brute est voisine de celle des blés durs. La torréfaction détruit $85 \mathrm{p}$. Ioo des inhibiteurs de l'arachide. La constante de dissociation apparente des complexes trypsine-inhibiteur est très faible, de l'ordre de $\mathrm{IO}^{-8} \mathrm{~mole} /$ litre. Elle diffère peu pour les différents blés sauf pour la variété Langue$d o c$, ainsi que pour l'arachide, où elle est trois à quatre fois plus faible.

\section{TABLEAU 2}

Inhibiteurs trypsiques des divers substrats

\begin{tabular}{|c|c|c|c|}
\hline \multirow{2}{*}{ Substrats } & \multicolumn{2}{|c|}{ Concentration : $10^{-\theta}$ mole* par gramme } & \multirow{2}{*}{$\begin{array}{c}\text { Cte de dissociation apparente } \\
\text { des complexes } \\
\text { trypsine-inhibiteurs : } \\
10^{-8} \text { mole* par litre }^{*}\end{array}$} \\
\hline & de produit & d'azote du produit & \\
\hline $\begin{array}{l}\text { Blés durs : } \\
\quad \text { variêté hybride } \ldots \ldots \ldots \ldots \ldots \ldots \\
\text { variété Lakota } \ldots \ldots \ldots \ldots \ldots\end{array}$ & $\begin{array}{l}10,7 \\
11,2\end{array}$ & $\begin{array}{l}631 \\
660\end{array}$ & $\begin{array}{l}6,0 \\
6,0\end{array}$ \\
\hline $\begin{array}{l}\text { Blés tendres : } \\
\quad \text { variété Étoile de Choisy } \ldots \ldots \ldots \\
\text { variété Languedoc } \ldots \ldots \ldots \ldots\end{array}$ & $\begin{array}{l}18,2 \\
27,5\end{array}$ & $\begin{array}{l}1240 \\
1840\end{array}$ & $\begin{array}{l}4,8 \\
1,9\end{array}$ \\
\hline $\begin{array}{l}\text { Farine d'arachide délipidée à } 80 \% \\
\text { brute } \ldots \ldots \ldots \ldots \ldots \ldots \ldots \ldots \ldots \ldots \ldots \ldots \ldots\end{array}$ & $\begin{array}{c}39 \\
5,7\end{array}$ & $\begin{array}{r}510 \\
74\end{array}$ & $\begin{array}{l}2,2 \\
2,3\end{array}$ \\
\hline Gluten & 0 & & \\
\hline Muscle de bœuf & 0 & & \\
\hline Lait & 0 & & \\
\hline
\end{tabular}

* On admet pour la trypsine pure une activité de 3500 unités National Formulary par mg et une masse molaire de 24000 .

Après vérification de l'homogénéité de leur variance par le test de Bartlett, les résultats obtenus pour la protéolyse trypsique des divers substrats ont été soumis à 1'analyse de variance, et la signification statistique des comparaisons entre moyennes, évaluée d'après le test de Fisher-Snedecor. Les calculs ont été faits sur Programma Ior (Olivetti) avec les programmes de Lowy et Manchon (I968-I969). Les différences sont considérées comme très significatives pour un seuil de probabilité $\mathrm{P}<0, \mathrm{OI}$, 


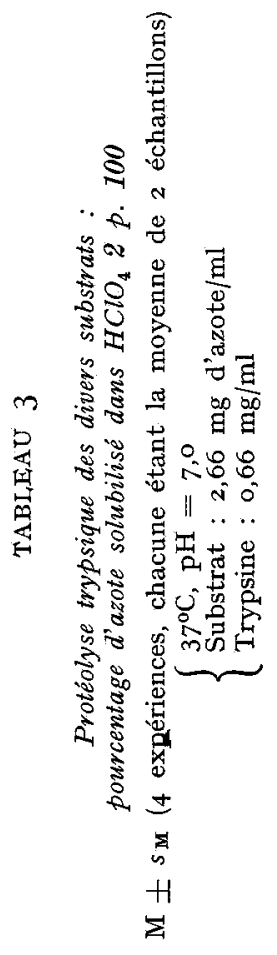

\begin{tabular}{|c|c|c|c|c|c|c|c|c|c|}
\hline \multirow{4}{*}{ 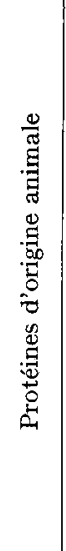 } & \multirow{2}{*}{$\begin{array}{l}\stackrel{0}{0} \\
\stackrel{\mathscr{0}}{0} \\
.00\end{array}$} & \multicolumn{2}{|c|}{ 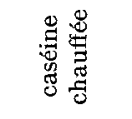 } & 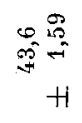 & 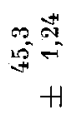 & 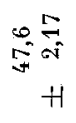 & 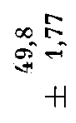 & 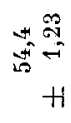 & 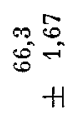 \\
\hline & & \multicolumn{2}{|c|}{ 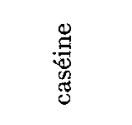 } & $\begin{array}{r}+1 \\
+1 \\
+1\end{array}$ & 㸃 & 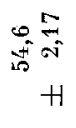 & 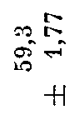 & 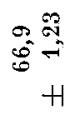 & $\begin{array}{r}0 \\
0 \\
0 \\
0 \\
0\end{array}$ \\
\hline & \multirow{2}{*}{ 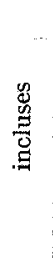 } & \multicolumn{2}{|c|}{ 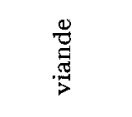 } & 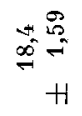 & 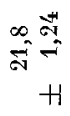 & 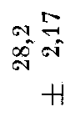 & $\stackrel{5}{8}$ & 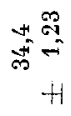 & 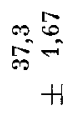 \\
\hline & & \multicolumn{2}{|c|}{ 节 } & 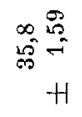 & 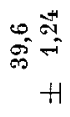 & 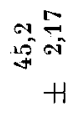 & 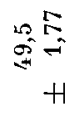 & 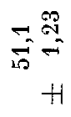 & 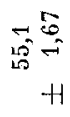 \\
\hline \multirow{8}{*}{ 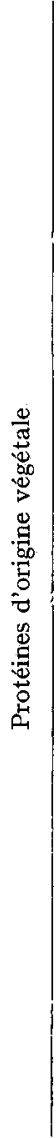 } & \multirow{2}{*}{ 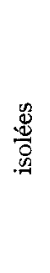 } & \multicolumn{2}{|c|}{ 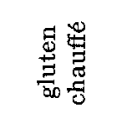 } & 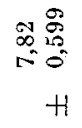 & 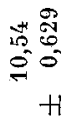 & 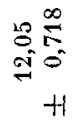 & 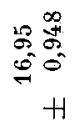 & 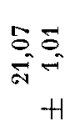 & 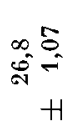 \\
\hline & & \multicolumn{2}{|c|}{ 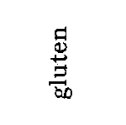 } & 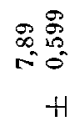 & 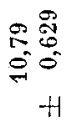 & 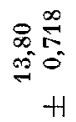 & 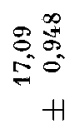 & $\begin{aligned} 0 & \bar{s} \\
\text { बे } & = \\
& H\end{aligned}$ & $\begin{array}{l}0 \\
+\infty \\
+\infty\end{array}$ \\
\hline & \multirow{6}{*}{$\begin{array}{l}0 \\
0 \\
0 \\
0 \\
0 \\
.0 \\
.0\end{array}$} & \multirow{2}{*}{ 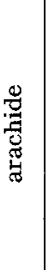 } & 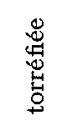 & \begin{tabular}{rl}
8 & 8 \\
8 & 8 \\
\hdashline & 0 \\
& $H$ \\
& $H$
\end{tabular} & 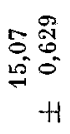 & 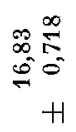 & 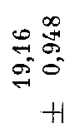 & 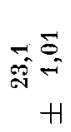 & 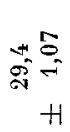 \\
\hline & & & 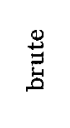 & 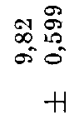 & 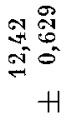 & 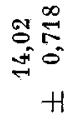 & 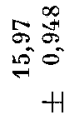 & $\begin{aligned} \stackrel{5}{0} \\
\stackrel{0}{\infty}=- \\
+\end{aligned}$ & $\stackrel{5}{=}$ \\
\hline & & \multirow{2}{*}{ 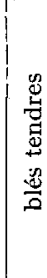 } & $\begin{array}{l}\text { हूँ } \\
\text { है } \\
\text { है } \\
\text { ज्ञ }\end{array}$ & 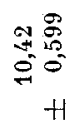 & 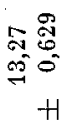 & 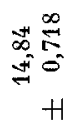 & 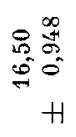 & 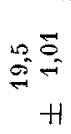 & 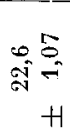 \\
\hline & & & 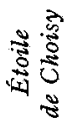 & 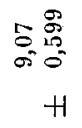 & $\begin{aligned} & \begin{array}{l}8 \\
0\end{array} \\
= & 0 \\
& 0 \\
& H\end{aligned}$ & 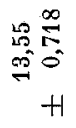 & 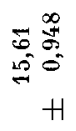 & $\begin{aligned} & \overline{0} \\
& \infty \\
& = \\
& H\end{aligned}$ & $\frac{5}{-5}$ \\
\hline & & \multirow{2}{*}{ 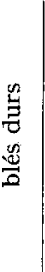 } & 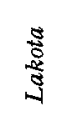 & 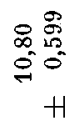 & 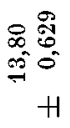 & 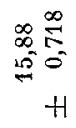 & 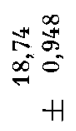 & $\begin{aligned} & \overrightarrow{0} \overrightarrow{0} \\
&= \\
&+\end{aligned}$ & 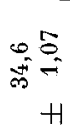 \\
\hline & & & $\begin{array}{l}\text { ज्ञ } \\
\text { ड़े } \\
\text { जे }\end{array}$ & 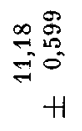 & 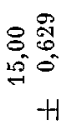 & $\begin{array}{r}\stackrel{\infty}{\stackrel{\infty}{\sigma}} \\
\stackrel{0}{=} \\
H\end{array}$ & 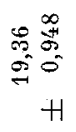 & 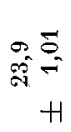 & 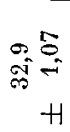 \\
\hline & \multicolumn{3}{|c|}{ 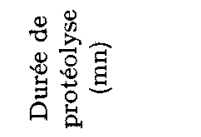 } & 18 & $\stackrel{ }{\circ}$ & 12 & $\stackrel{\varnothing}{\circ}$ & 8 & ฐิ \\
\hline
\end{tabular}



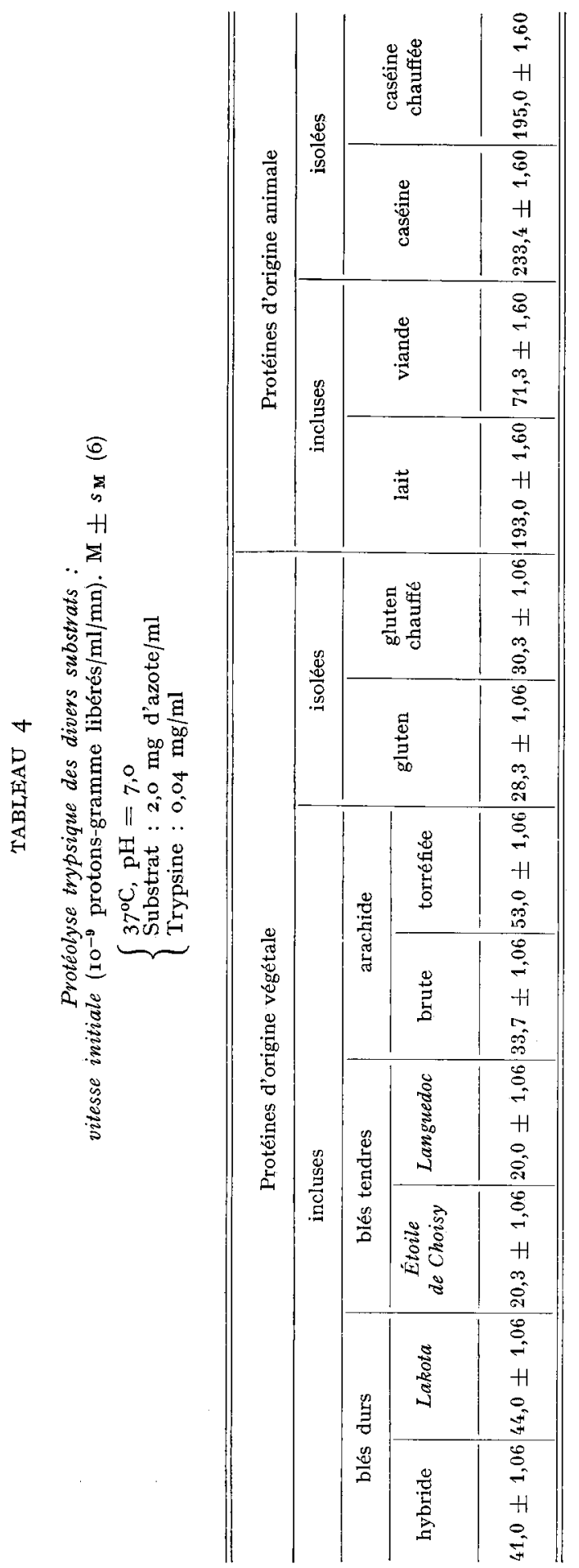
significatives pour 0 , or $<\mathrm{P}<0,05$, non significatives pour $\mathrm{P}>0,05$. Le tableau 3 donne la valeur de la protéolyse déterminée par dosage de l'azote solubilisé dans l'acide perchlorique. On constate que la protéolyse des substrats d'origine animale est supérieure à celle des substrats d'origine végétale. Sa valeur décroît dans l'ordre : caséine, lait, viande, blés durs, (blés tendres, gluten, arachide) $(\mathrm{P}<0,0 \mathrm{r})$. La différence entre les trois derniers substrats ainsi qu'entre blés de même espèce est très faible $(\mathrm{P}>0,05)$. Après chauffage à I00 ${ }^{\circ} \mathrm{C}$ pendant $\mathrm{I} 5$ minutes, la protéolyse de la caséine est moindre $(\mathrm{P}<0, \mathrm{or})$ et voisine de celle du lait alors que celle du gluten est inchangée $(P>0,05)$. Après torréfaction, celle de 1'arachide est augmentée $(\mathrm{P}<0,0 \mathrm{I})$. Les tableaux 4 et 6 donnent la vitesse initiale de la protéolyse déterminée

\section{TABLEAU 5}

Conditions de la protéolyse pepsique solubilisant dans $\mathrm{HClO}_{4} \& \mathrm{p} .100$

$45 \mathrm{p} .100$ de l'azote des divers substrats

$37^{\circ} \mathrm{C}, \mathrm{pH}=1,9$

Substrat : 2,o mg d'azote/ml

\begin{tabular}{|c|c|c|c|c|c|}
\hline \multicolumn{4}{|c|}{ Substrats } & $\begin{array}{l}\text { Concentration } \\
\text { de pepsine }\end{array}$ & $\begin{array}{l}\text { Durée de } \\
\text { protéolyse }\end{array}$ \\
\hline \multirow{5}{*}{$\begin{array}{l}\text { Protéines } \\
\text { d'origine } \\
\text { végétale }\end{array}$} & \multirow{3}{*}{ Incluses } & Blés durs & $\begin{array}{l}\text { hybride } \\
\text { Lakota }\end{array}$ & $\begin{array}{l}0,48 \\
0,48\end{array}$ & $\begin{array}{l}60 \\
60\end{array}$ \\
\hline & & Blés tendres & $\begin{array}{l}\text { Étoile de Choisy } \\
\text { Languedoc }\end{array}$ & $\begin{array}{l}0,48 \\
0,48\end{array}$ & $\begin{array}{l}60 \\
60\end{array}$ \\
\hline & & Arachide & $\begin{array}{l}\text { Brute } \\
\text { Torréfiée }\end{array}$ & $\begin{array}{l}0,48 \\
0,48\end{array}$ & $\begin{array}{r}120 \\
30\end{array}$ \\
\hline & \multirow{2}{*}{ Isolées } & \multicolumn{2}{|c|}{ Gluten } & 0,03 & 120 \\
\hline & & \multicolumn{2}{|c|}{ Gluten chauffé } & 0,03 & 120 \\
\hline \multirow{4}{*}{$\begin{array}{l}\text { Protéines } \\
\text { d'origine } \\
\text { animale }\end{array}$} & \multirow{2}{*}{ Incluses } & \multicolumn{2}{|c|}{ Lait } & 0,66 & 15 \\
\hline & & \multicolumn{2}{|c|}{ Viande } & 0,66 & 5 \\
\hline & \multirow{2}{*}{ Isolées } & \multicolumn{2}{|c|}{ Caséine } & 0,03 & $60^{\prime}$ \\
\hline & & \multicolumn{2}{|c|}{ Caséine chauffée } & 0,03 & 60 \\
\hline
\end{tabular}

par dosage des protons libérés avant et après protéolyse pepsique. Avant protéolyse pepsique, les résultats sont semblables aux précédents. Après une même protéolyse pepsique, la supériorité de la protéolyse trypsique des substrats d'origine animale subsiste, bien qu'atténuée. Mais il n'y a plus de différence entre substrats de même origine, végétale ou animale, sauf pour l'arachide torréfiée qui est alors voisine de ces derniers. 

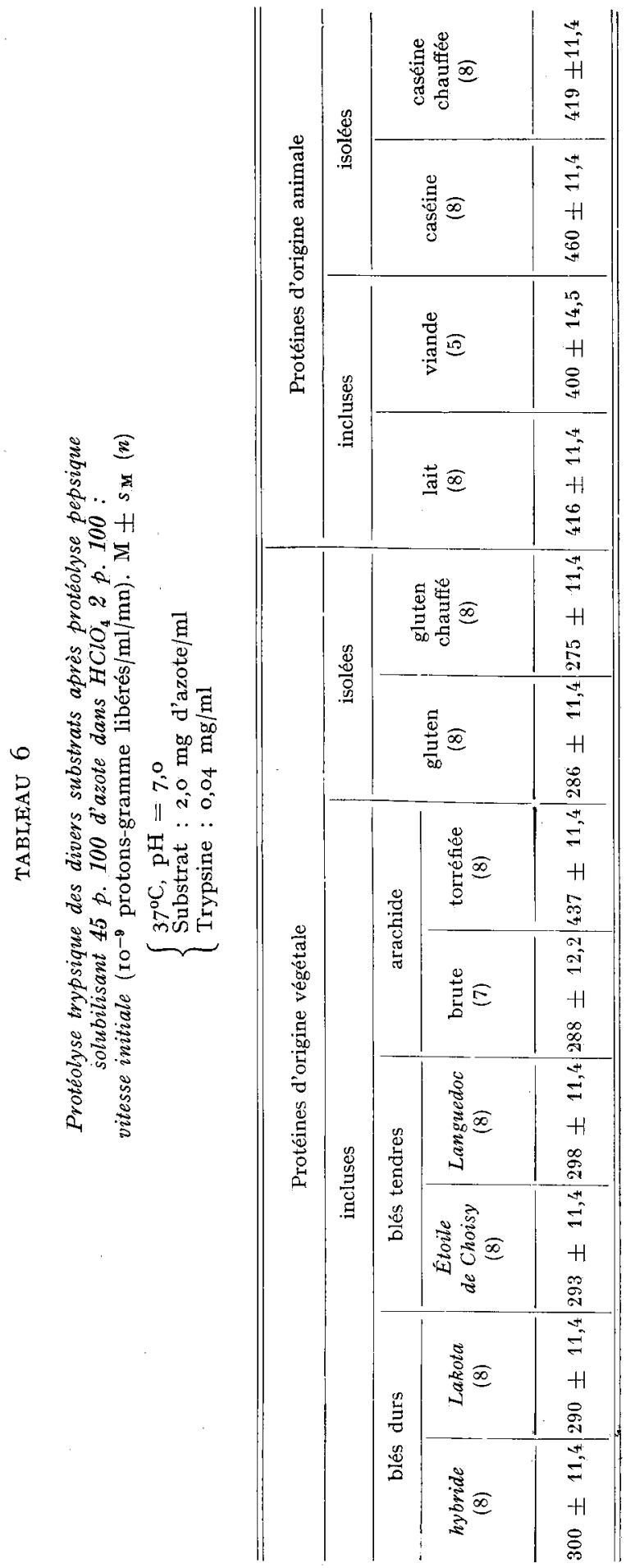


\section{DISCUSSION}

Les deux méthodes utilisées établissent la même hiérarchie pour la protéolyse trypsique des divers substrats. Cependant, par la méthode de dosage des protons où la concentration d'enzyme est la plus faible, les différences sont plus grandes, surtout pour les substrats d'origine végétale. Ceci pourrait être dû à leurs teneurs différentes en inhibiteurs trypsiques neutralisant une fraction non négligeable d'enzyme. En effet, la protéolyse trypsique des blés tendres et de l'arachide brute est respectivement plus faible que celle des blés durs et de l'arachide torréfiée, qui contiennent moins d'inhibiteurs. BIELORAI (I969), constate de même après torréfaction de l'arachide une moindre teneur en inhibiteurs corrélative à une augmentation de sa protéolyse trypsique. Cependant, pour une même teneur en inhibiteurs rapportée à l'azote, la protéolyse des blés durs est supérieure à celle de l'arachide brute, et il n'y a aucune différence entre les protéolyses des blés tendres et de leur gluten exempt d'inhibiteurs. Leur teneur différente en inhibiteurs ne peut donc expliquer leur différence de protéolyse qui doit dépendre de la structure de leurs protéines.

La tryspine ne rompant que les liaisons peptidiques où est engagé le carboxyle de la lysine ou de l'arginine, la teneur en ces deux acides aminés (AITMAN et DitTMER, I968; Busson et al., I966), pourrait intervenir. Elle ne semble cependant pas être en relation évidente avec la protéolyse trypsique des divers substrats. De plus, l'action préalable d'un traitement non drastique respectant la lysine et l'arginine peut modifier la protéolyse trypsique. Ainsi, nous observons une protéolyse de la caséine, moindre après chauffage modéré, de même que SwIRSKI et al. (Ig63) avec la caséine entière et la caséine $\alpha$. Par contre, comme pour la pepsine (CAMUs et SAUTIER, 1972), la résistance à la trypsine se maintient après chauffage du gluten. Après torréfaction, la protéolyse trypsique de l'arachide est augmentée. Selon AdRIAN (1967), une torréfaction modérée n'agirait pas sur la lysine. Mais, comme dans le lait, elle serait bloquée ou détruite par un traitement plus drastique. L'action favorable d'une protéolyse pepsique préalable pourrait résulter d'une meilleure accessibilité par la trypsine des liaisons peptidiques spécifiques. Ce serait donc leur disponibilité plus que les teneurs en lysine et en arginine, qui interviendrait.

Étant donné la tendance à l'égalisation des protéolyses trypsiques des divers substrats après action de la pepsine, les différences observées lors de la seule protéolyse trypsique pourraient paraître de moindre intérêt physiologique. Cependant, la protéolyse pepsique préalable, qui, ici, a été arrêtée à un même stade de dégradation des divers substrats, requiert pour chacun d'eux des conditions très différentes, soit de concentration d'enzyme, soit de temps d'action. Or, in vivo, ce même stade de protéolyse pepsique n'est peut être pas atteint pour tous les substrats, surtout lors de l'ingestion simultanée de plusieurs d'entre eux. Il serait donc important de connaître les conditions physiologiques de la protéolyse pepsique ( $\mathrm{pH}$, concentration en pepsine, temps de séjour gastrique) et l'état de dégradation de divers substrats à leur sortie de l'estomac.

D'autre part, nous n'avons pas pris en considération la granulométrie des produits étudiés, qui pourrait, peut-être, intervenir dans la hiérarchie de nos résultats. 


\section{SUMMARY}

\section{IN VIVO TRYPTIC PROTEOLYSIS OF VARIOUS FOODS}

The time course of tryptic proteolysis of various foods (wheat, groundnut, meat, milk, casein, wheat gluten) was determined by measuring perchloric acid soluble nitrogen and released protons. The trypsin inhibitors content of these materials was also determined. Proteolysis was faster and more complete with the substrates of animal origin, and decreased in the following order: casein, milk, meat, Triticum durum, Triticum vulgare, gluten, and groundnut, the differences between the last three being very small. Heating at $100^{\circ} \mathrm{C}$ for $15 \mathrm{mn}$ decreased proteolysis of casein but not of gluten. After peptic proteolysis, there was no difference between substrates of the same origin. However, animal foods were still better substrates. Trypsin inhibitors were found in wheat and groundnut only. The inhibitors content of groundnut was two and four fold higher than those of Triticum durum and Triticum vulgare, respectively. The complex structure of the proteins of various foods seemed to play a more important part than their trypsin inhibitors and lysine and arginine content.

\section{RÉFÉRENCES BIBLIOGRAPHIQUES}

Adrian J., I967. La réaction de Maillard. IV. Étude du comportement de quelques acides aminés au cours du grillage d'aliments protidiques. Ann. Nutr. Alim., 21, I29-I47.

Aitman P. L., Dittmer D. S., I968. Metabolism, 53-59, Fed. amer, soc. exper. biol, Bethesda.

Bielorai R., rg68. Comparative digestibility of groundnut and soyabean meal in vitro and in chicks. J. Sci. food A gric., 20, 345-348.

Busson F., Fauconneau G., Pion R., Montreuil J., r966. Protides, $2^{\mathrm{e}}$ section : acides aminés. Ann. Nutr. Alim., 20, I99-2I9.

CAMUS M.-C., Sautier C., I972. Protéolyse pepsique d'aliments d'origine animale et végétale. Ann. Biol. anim., Bioch. Biophys., 12, $28 \mathrm{I}-288$.

LAPORTE J. C., I964. Mise en évidence et étude de facteurs antitrypsiques dans les céréales. Thèse Sciences $3^{\mathbf{e}}$ cycle, Paris.

Lowy R., Manchon P., I968-I969. Éléments de statistiques appliqués à la biologie, Olivetti, Paris.

National Formulary, 1970. 746-747, American Pharmaceutical Association, Washington.

Pusztal A., 1967. Trypsin inhibitors of plant origin, their chemistry and potential role in animal nutrition. Nutr. Abst. Rev., 37, I-9.

Swirski M., Allouf R., Cheftel H., r963. Contribution à l'étude de la chaleur sur les caséines. Bull. Soc. Chim. Biol., 45, g01-911.

YoN J., r963. Étude des réactions enzymatiques. In LoISELEUR J., Techniques de laboratoire, T. I, Fasc. 2, 936-959, $3^{\text {e }}$ ed., Masson, Paris. 